\title{
Thermodynamics of RNA melting, one base pair at a time
}

\author{
EVGENIA N. NIKOLOVA and HASHIM M. AL-HASHIMI \\ Chemical Biology Doctoral Program, Department of Chemistry and Biophysics, University of Michigan, Ann Arbor, Michigan 48109, USA
}

\begin{abstract}
The melting of base pairs is a ubiquitous feature of RNA structural transitions, which are widely used to sense and respond to cellular stimuli. A recent study employing solution nuclear magnetic resonance (NMR) imino proton exchange spectroscopy provides a rare base-pair-specific view of duplex melting in the Salmonella FourU RNA thermosensor, which regulates gene expression in response to changes in temperature at the translational level by undergoing a melting transition. The authors observe "microscopic" enthalpy-entropy compensation-often seen "macroscopically" across a series of related molecular speciesacross base pairs within the same RNA. This yields variations in base-pair stabilities that are an order of magnitude smaller than corresponding variations in enthalpy and entropy. A surprising yet convincing link is established between the slopes of enthalpyentropy correlations and RNA melting points determined by circular dichroism (CD), which argues that unfolding occurs when base-pair stabilities are equalized. A single AG-to-CG mutation, which enhances the macroscopic hairpin thermostability and folding cooperativity and renders the RNA thermometer inactive in vivo, spreads its effect microscopically throughout all base pairs in the RNA, including ones far removed from the site of mutation. The authors suggest that an extended network of hydration underlies this long-range communication. This study suggests that the deconstruction of macroscopic RNA unfolding in terms of microscopic unfolding events will require careful consideration of water interactions.
\end{abstract}

Keywords: NMR imino proton exchange; base-pair thermodynamics; RNA folding and melting; RNA thermosensors; riboswitches

The melting and forming of RNA duplex structure are reoccurring structural transitions that are used in a variety of functional contexts. For example, transient short hairpins can direct RNA cotranscriptional folding by masking elements prone to kinetic traps and releasing them via melting transitions once downstream elements involved in long-range native contacts are transcribed (Nagel and Pleij 2002). Duplex melting, in general catalyzed by protein chaperones (Herschlag 1995) or induced by competing trans-acting small RNAs (Kozak 2005), is often a requirement for rescuing RNA from kinetic traps (Treiber and Williamson 2001) and allowing transformations to occur between functionally different RNA structures (Al-Hashimi and Walter 2008). The melting of duplex mRNA prior to ribosome entry is believed to provide a general mechanism for periodically stalling translation and promoting proper cotranslational protein folding and other processes (Kozak 2005; Watts et al. 2009). While melting transitions have

Reprint requests to: Hashim M. Al-Hashimi, Chemical Biology Doctoral Program, Department of Chemistry and Biophysics, $930 \mathrm{~N}$. University Avenue, University of Michigan, Ann Arbor, MI 48109, USA; e-mail: hashimi@umich.edu; fax: (734)647-4865.

Article published online ahead of print. Article and publication date are at http://www.rnajournal.org/cgi/doi/10.1261/rna.2235010. been studied for decades using a variety of biophysical techniques, most studies have relied on bulk spectroscopic measurements such as UV absorbance, circular dichroism (CD), and calorimetry (Jaeger et al. 1993), which typically provide a macroscopic view of melting that fails to resolve potential differences in the melting behavior of individual base pairs. By measuring the thermodynamic stability of individual base pairs in an RNA thermosensor and an inactive mutant, Schwalbe and co-workers (Rinnenthal et al. 2010) provide a rare site-specific view of duplex melting and revive the power of a long-standing nuclear magnetic resonance (NMR) technique to study RNA unfolding at atomic resolution.

The target of their studies is the temperature labile Salmonella FourU RNA thermometer, which regulates gene expression in response to changes in temperature by sequestering the Shine-Dalgarno ribosomal-binding sequence within a hairpin at low temperatures and liberating it via a melting transition at high temperatures. The AU-rich hairpin structure also contains several noncanonical base pairs that are believed to confer its reduced thermostability. Also examined is a single-point mutant (A8C) that exhibits a repressed phenotype in vivo, in which an AG mismatch within the stem-loop structure is replaced with a CG Watson-Crick (WC) base pair. 
The NMR experiments take advantage of the fact that relatively acidic imino protons of base-paired guanines and uridines/thymines $\left(\mathrm{p} K_{\mathrm{a}} \approx 9.2-9.6\right)$ can exchange with basic solvents when they melt to adopt an "open" conformation but not when they are in a "closed," intrahelical conformation. By measuring the exchange rate $\left(k_{\mathrm{ex}}\right)$ of single imino protons with solvent, it is possible to probe the opening/closing of individual base pairs, and deduce lifetimes of the closed and open states and the kinetics of their interconversion. This NMR technique is not new in its basic principle. Crothers (Crothers et al. 1974), Patel (Patel and Hilbers 1975), and their respective coworkers used one-dimensional (1D) NMR imino proton line broadening back in the early 1970 's to estimate thermodynamic parameters associated with helical melting and end-fraying in tRNA and short DNA duplexes. Subsequent developments in NMR pulse sequences and their application under variable base catalyst concentration and temperature allowed for a direct quantitative measurement of proton exchange rates and the elucidation of a generalized two-step exchange mechanism in nucleic acids by Leroy and Guéron (Leroy et al. 1985, 1988; Gueron et al. 1987) involving a single base-pair opening mode. In this mechanism, transient and independent base-pair opening is followed by direct, or water-mediated, imino proton transfer to a proton acceptor (Fig. 1A). The acceptor can be either an external base catalyst (e.g., $\mathrm{NH}_{3}$ or phosphate) or an intrinsic catalystnitrogen from the opposite nucleobase ( $\mathrm{N} 1$ of $\mathrm{A}$ or $\mathrm{N} 3$ of $\mathrm{C})$ in the oligonucleotide- that has unfavorably low $\mathrm{p} K_{\mathrm{a}}(3.7$ or 4.2$)$ but high local concentration (Gueron and Leroy 1995). This framework allowed Leroy and Guéron (Leroy et al. 1988) to accurately quantify site-specific base-pair lifetimes and activation enthalpies in DNA and subsequently in RNA by Varani and coworkers (Leroy et al. 1988; Varani et al. 1989). Current NMR methodology allows measurement of intrinsic exchange times ranging between $\sim 1 \mathrm{msec}$ and $2 \mathrm{sec}$ using relaxation techniques, and longer than $\sim 2$ min using real-time $\mathrm{H}-\mathrm{D}$ exchange kinetics, while the window in between is currently inaccessible to NMR (Gueron and Leroy 1995).

In their study, Rinnenthal et al. (2010) measured imino proton exchange rates using so-called magnetization trans- fer-type NMR experiments under EX2 conditions. Here, the catalyst (phosphate) concentration is kept sufficiently low such that base-pair opening/closing rapidly equilibrates prior to solvent transfer (Gueron and Leroy 1995), but is kept sufficiently high such that imino proton exchange is still dominated by the external base (i.e., dependent on added catalyst), and catalysis by nucleobase partners can be neglected. This regime circumvents time-consuming catalyst titrations and avoids undesired effects of high base concentration on nucleic acid structure. The optimal conditions required to meet these criteria will vary with catalyst and RNA and could be verified for a subset of peaks by measuring exchange rates in the 1D mode as a function of catalyst concentration (low to moderate) and, if conducting 
a temperature dependence, at low and high temperatures. In this time-saving manner, exchange rates can be used to deduce the equilibrium constant, $K_{\text {diss }}$, for base-pair opening $\left(K_{\mathrm{diss}}=k_{\mathrm{op}} / k_{\mathrm{cl}}\right)$ (see Fig $\left.1 \mathrm{~A}\right)$. In the NMR experiment, water magnetization is initially selectively inverted through the application of a $180^{\circ}$ soft pulse. The "magnetically" labeled water protons are then allowed to exchange with the imino protons for a variable mixing period, $\tau_{\mathrm{m}}$, and acquisition pulses are then used to record a two-dimensional heteronuclear single-quantum correlation (2D HSQC) imino proton/nitrogen spectrum. Because the water magnetization is inverted relative to the imino protons, exchange causes a well-established modulation in the apparent imino proton signal. By measuring that signal as a function of different $\tau_{\mathrm{m}}$ delays, one can directly extract the apparent exchange rate constant $\left(k_{\text {ex }}\right)$, which depends on the exchange rate from the open conformation $\left(k_{\mathrm{ex}, \text { open }}\right)$, measured in mononucleotides mimicking the open state, and the equilibrium dissociation constant ( $\left.K_{\text {diss }}\right)$ (Fig. 1A). By measuring exchange rates (and $K_{\text {diss }}$ ) as a function of temperature, Rinnenthal et al. (2010) were further able to deduce all thermodynamic parameters for base-pair opening, including free energy $\left(\Delta G_{\text {diss }}\right)$, enthalpy $\left(\Delta H_{\text {diss }}\right)$, and entropy $\left(\Delta S_{\text {diss }}\right)$ differences for multiple base pairs in the Salmonella FourU RNA thermosensor and its thermostable A8C mutant. Alternatively, more exhaustive catalyst titrations using the $2 \mathrm{D}$ imino proton exchange experiment could be used to further extract the individual rate constants for base-pair opening $\left(k_{\mathrm{op}}\right)$ and closing $\left(k_{\mathrm{cl}}\right)$ and the corresponding free energy, enthalpy, and entropy of activation.

At temperatures below the melting temperature $\left(T_{\mathrm{m}}\right)$, Rinnenthal et al. (2010) observed a broad distribution of base-pair opening free energies $\left(\Delta G_{\text {diss }}\right)$, particularly for the mutant sequence, with base pairs near the terminal ends and loops tending to favor melting. The variations in enthalpy $\left(\Delta H_{\text {diss }}\right)$ and entropy $\left(\Delta S_{\text {diss }}\right)$ are an order of magnitude larger, but they compensate one another to yield much smaller differences in $\Delta G_{\text {diss }}$ (Fig. 1B,C). The differences in $\Delta G_{\text {diss }}$ diminish steadily with increasing temperature and, remarkably, coalesce to a common value precisely at the melting temperature $\left(T_{\mathrm{m}}\right)$, obtained independently from CD (Fig. 1D,E). These data are particularly convincing for the mutant sequence, for which there are more data points and a broader distribution of $\Delta G_{\text {diss }}$ values at low temperatures. This suggests that cooperative global melting occurs only when all base pairs have comparable stabilities. Notably, the coalescence $\Delta G_{\text {diss }}$ value is not zero but rather slightly positive. Rinnenthal et al. (2010) explain this by residual stacking between neighboring nucleobases associated with the open state when cooperative base-pair melting is considered $(\sim 10 \mathrm{~kJ} /$ $\mathrm{mol}$ ), which remains undetected at low temperatures in which base-pair opening is primarily uncorrelated. Interestingly, the single AG-to-CG mutation results in an $\sim 12^{\circ}$ higher $T_{\mathrm{m}}$ value, as measured by $\mathrm{CD}$, and a correspondingly higher $\Delta G_{\text {diss }}$ coalescence temperature for all base pairs, including ones that are several residues away from the mutation site (Fig. 1D,E). A shift to slightly positive $\Delta G_{\text {diss }}$ at the intersection point could potentially arise from a systematic bias in the exchange measurements (i.e., determination of $k_{\text {ex,open }}$ for nucleotide triphosphates) or thermodynamic assumptions. Such would be the case if the unknown factor $\alpha$, which enters the apparent $K_{\text {diss }}$ or $k_{\text {ex,open }}$ as a coefficient and which reflects hindered accessibility and/ or electrostatic effects in the interaction between the catalyst and the open RNA base compared with mononucleotides, is not unity as commonly assumed. However, values for $\alpha$ of the phosphate catalyst would have to be reduced by approximately two orders of magnitude to solely justify the shift in coalescence $\Delta G_{\text {diss }}$, which would contradict previous studies reporting a relatively small spread of $\alpha K_{\text {diss }}(<10 \times)$ measured with catalysts of different sizes and charges (Leroy et al. 1985; Kochoyan et al. 1988).

Since $\Delta H_{\text {diss }}$ and $\Delta S_{\text {diss }}$ are assumed to be independent of temperature, the observed behavior implies that variations in $\Delta H_{\text {diss }}$ across base pairs are linearly compensated by changes in $\Delta S_{\text {diss }}$, such that $\Delta G_{\text {diss }}$ converges to a common value at a compensatory temperature $T_{\mathcal{c}}$, which is equal to $T_{\mathrm{m}}$. While an excellent linear $\Delta H_{\text {diss }}\left(\Delta S_{\text {diss }}\right)$ correlation is observed for both the wild-type (wt) and mutant sequences, such correlations should in general be treated with caution, particularly when a narrow range of $\Delta G_{\text {diss }}$ is observed relative to the spread of $\Delta H_{\text {diss }}$, as is the case here, from which $\Delta G_{\text {diss }}=\Delta H_{\text {diss }}-T \Delta S_{\text {diss }} \approx$ const. naturally implies a linear relationship and/or when $\left|\Delta G_{\text {diss }}\right|<\left|\Delta H_{\text {diss }}\right|$ with $\Delta S_{\text {diss }}$ calculated by subtraction of the independently measured free energy and enthalpy (Lumry and Rajender 1970; Sharp 2001). There, large correlated errors in $\Delta H_{\text {diss }}$ and $\Delta S_{\text {diss }}$ could produce an artificial linear relationship between the two with high correlation coefficients. However, for this study, the correlations appear to be real because the data satisfy a statistical test for error compensation effects (Sharp 2001); the slope of the correlation for the wt and mutant RNAs are different and in both cases coincident with $T_{\mathrm{m}}$, and the $\Delta G_{\text {diss }}(T)$ van't Hoff plots for multiple residues seemingly intersect at a common point. As would be expected for an intrinsic correlation, the slope represents a temperature at which variations in entropy and enthalpy are balanced, and this appears to coincide with $T_{\mathrm{m}}$ for cooperative duplex melting. Thus, the melting thermodynamics of various base pairs in the RNA seem to be enslaved along a predefined $\Delta H_{\text {diss }}\left(\Delta S_{\text {diss }}\right)$ line.

What might be the common thread that links the thermodynamics of different base pairs? Enthalpy-entropy compensation has been observed in a variety of biological systems and is often linked to interactions with water. For example, early studies on small molecules and proteins in aqueous solution have shown that the slopes of $\Delta H_{\text {diss }}\left(\Delta S_{\text {diss }}\right)$ cluster consistently around a common temperature of $\sim 280 \mathrm{~K}$ for widely different processes, and thus 
could reflect a common solvent property (Lumry and Rajender 1970). Solvent interactions have also been invoked to explain enthalpy-entropy compensation during protein unfolding (Liu et al. 2000) and base-opening in imino proton exchange studies of a DNA dodecamer (Chen and Russu 2004). Typically, the effect is observed macroscopically across related systems, for example, in comparing binding energies of related ligands with target receptors (Breslauer et al. 1987; Lis and Sharon 1998) or the unfolding energies of different proteins (Liu et al. 2000), and not microscopically within a given system as observed here for the melting of individual base pairs within the same RNA. Indeed, the observation of enthalpy-entropy compensation within different base pairs supports the Tinoco-Uhlenbeck approximation of decomposing nucleic acid helices into independent base-pair units with nearestneighbor effects (Tinoco et al. 1971). This long-standing link between solvent and enthalpy-entropy compensation, together with the large $\Delta H_{\text {diss }}$ and $\Delta S_{\text {diss }}$ values derived, which rule out an intrinsic RNA effect, lead Rinnenthal et al. (2010) to invoke interactions with water as the driving force for the observed enthalpy-entropy compensation. Rinnenthal et al. (2010) suggested that base-pair opening likely results in the release of highly ordered water molecules from the major groove, giving rise to a loss of solvent enthalpy that is compensated for by a gain in solvent entropy, and that these opposing contributions are larger for GC versus AU base pairs. They speculated that the AG mismatch is responsible for destabilizing its neighbors and, in turn, the overall helix through solventmediated communication with remote sites. They concluded that melting of the water shell should precede RNA melting, at which point favorable RNA-solvent interactions on duplex stability should be neutralized.

There is ample precedence for such a role of solvent in RNA structural stability. Molecular dynamics (MD) simulations by Auffinger and Westhof (2002) reveal a "premelting" phase due to water shell disruption before global RNA duplex melting. MD simulations also suggest that DNA base-opening induces water shell perturbations (Giudice et al. 2003). Biophysical studies using neutron scattering provide evidence that hydrated RNA undergoes a dynamic glass transition that is driven by solvent interactions, much like hydrated proteins (Caliskan et al. 2006). Analysis of RNA crystal structures and MD simulations reveals highly specific AG and GU hydration pockets with long-lived waters and cooperative contacts with neighboring water/ RNA groups (Auffinger and Westhof 1997; Reblova et al. 2003).

Thus, it is possible that local differences in conformation and respective hydration patterns between wt and mutant RNA that are somehow transduced down the helix to remote sites affect the relative $\Delta H_{\text {diss }}$ and $\Delta S_{\text {diss }}$ values for individual base pairs as well as increase the overall thermostability of the mutant hairpin. Diminished stacking interactions in the mismatched duplex are used to explain the dramatically lower cooperativity of the wt RNA melting transition. Single mismatched GA base pairs are known to destabilize nucleic acid duplexes (Aboul-ela et al. 1985) and reduce coaxial stacking relative to CG base pairs when placed at helical interfaces (Schroeder et al. 1996). Diminished stacking can arise from structural perturbations and conformational exchange between different base-pairing geometries reported in structural studies of GA mismatches in DNA and RNA (see Allawi and SantaLucia 1998). In fact, sensitivity to next-nearest neighbor context has been observed for RNA GA mismatch structures, arguing for potential long-range effects dictated by adjacent elements (Morse and Draper 1995). The lower cooperativity of the wt is consistent with single-molecule forcepulling experiments on DNA hairpins, which show that inclusion of various mismatches (TT, GA, GT, etc.) reduces the cooperativity of the unfolding transition due to appreciably populated intermediate, partially unfolded, states (Woodside et al. 2006). Thus, the thermodynamic stability of the RNA structure seems to be intimately linked to solvent interactions, which are very difficult to visualize using techniques such as X-ray crystallography and NMR spectroscopy.

As indicated by previous research, for some nucleobases, the dependence of $k_{\text {ex }}(T)$ with temperature deviates from the behavior expected based on the assumed two-state model and transition state theory. This could reflect temperatureinduced changes in the RNA structure (and therefore that $\Delta H$ and $\Delta S$ are not independent of temperature as assumed) and/or deviations in the assumption that base-pair opening is uncorrelated at higher temperatures near $T_{\mathrm{m}}$. Such effects can be interrogated in future studies by acquiring additional data to allow the fitting of more complicated statistical models. In addition, the precise nature of the "open" state probed by NMR exchange measurements, and whether it is the same state probed by bulk optical melting measurements, is yet to be established and indeed there are studies that suggest they could be different (Benight et al. 1988).

Through their study, Rinnenthal et al. (2010) have resurrected and modernized a long-standing NMR technique in obtaining site-specific information regarding the thermodynamic stability of RNA structure. Imino proton exchange is limited to specific base-pair opening transitions that occur with time constants in the range of approximately $1 \mathrm{msec}$ to 2 sec and, therefore, provides primarily a cross-sectional view of RNA stability. However, other advances in solution (Furtig et al. 2007; Zhang et al. 2007; Johnson and Hoogstraten 2008; Hansen et al. 2009; Bailor et al. 2010) and solid state (Olsen et al. 2010) NMR and other biophysical techniques (Al-Hashimi and Walter 2008) are making it possible to quantitatively describe microscopic structural transitions along the entire RNA structure taking place over timescales spanning picoseconds to several minutes. While it appears that the dissection of macroscopic RNA stability in terms of 
constituent microscopic contributions is within reach, the present study calls for new experimental tools for characterizing dynamic interactions between water and RNA. Perhaps there is yet another well-known NMR technique that we can draw on to achieve this goal.

\section{REFERENCES}

Aboul-ela F, Koh D, Tinoco I Jr, Martin FH. 1985. Base-base mismatches. Thermodynamics of double helix formation for dCA3XA3G + dCT3YT3G (X, Y = A,C,G,T). Nucleic Acids Res 13: 4811-4824.

Al-Hashimi HM, Walter NG. 2008. RNA dynamics: It is about time. Curr Opin Struct Biol 18: 321-329.

Allawi HT, SantaLucia J Jr. 1998. Nearest-neighbor thermodynamic parameters for internal $\mathrm{G} \bullet \mathrm{A}$ mismatches in DNA. Biochemistry 37: 2170-2179.

Auffinger P, Westhof E. 1997. RNA hydration: Three nanoseconds of multiple molecular dynamics simulations of the solvated tRNA ${ }^{\text {Asp }}$ anticodon hairpin. J Mol Biol 269: 326-341.

Auffinger P, Westhof E. 2002. Melting of the solvent structure around a RNA duplex: A molecular dynamics simulation study. Biophys Chem 95: 203-210.

Bailor MH, Sun X, Al-Hashimi HM. 2010. Topology links RNA secondary structure with global conformation, dynamics, and adaptation. Science 327: 202-206.

Benight AS, Schurr JM, Flynn PF, Reid BR, Wemmer DE. 1988. Melting of a self-complementary DNA minicircle. Comparison of optical melting theory with exchange broadening of the nuclear magnetic resonance spectrum. J Mol Biol 200: 377-399.

Breslauer KJ, Remeta DP, Chou WY, Ferrante R, Curry J, Zaunczkowski D, Snyder JG, Marky LA. 1987. Enthalpy-entropy compensations in drug-DNA binding studies. Proc Natl Acad Sci 84: 8922-8926.

Caliskan G, Briber RM, Thirumalai D, Garcia-Sakai V, Woodson SA, Sokolov AP. 2006. Dynamic transition in tRNA is solvent induced. J Am Chem Soc 128: 32-33.

Chen C, Russu IM. 2004. Sequence dependence of the energetics of opening of AT basepairs in DNA. Biophys J 87: 2545-2551.

Crothers DM, Cole PE, Hilbers CW, Shulman RG. 1974. The molecular mechanism of thermal unfolding of Escherichia coli formylmethionine transfer RNA. J Mol Biol 87: 63-88.

Furtig B, Buck J, Manoharan V, Bermel W, Jaschke A, Wenter P, Pitsch S, Schwalbe H. 2007. Time-resolved NMR studies of RNA folding. Biopolymers 86: 360-383.

Giudice E, Varnai P, Lavery R. 2003. Base-pair opening within B-DNA: Free energy pathways for GC and AT pairs from umbrella sampling simulations. Nucleic Acids Res 31: 1434-1443.

Gueron M, Leroy JL. 1995. Studies of base-pair kinetics by NMR measurement of proton exchange. Methods Enzymol 261: 383-413.

Gueron M, Kochoyan M, Leroy JL. 1987. A single mode of DNA basepair opening drives imino proton exchange. Nature 328: 89-92.

Hansen AL, Nikolova EN, Casiano-Negroni A, Al-Hashimi HM. 2009. Extending the range of microsecond-to-millisecond chemical exchange detected in labeled and unlabeled nucleic acids by selective carbon R(1rho) NMR spectroscopy. J Am Chem Soc 131: 3818-3819.

Herschlag D. 1995. RNA chaperones and the RNA folding problem. J Biol Chem 270: 20871-20874.

Jaeger JA, SantaLucia J Jr, Tinoco I Jr. 1993. Determination of RNA structure and thermodynamics. Annu Rev Biochem 62: 255-287.

Johnson JE Jr, Hoogstraten CG. 2008. Extensive backbone dynamics in the GCAA RNA tetraloop analyzed using ${ }^{13} \mathrm{C}$ NMR spin relaxation and specific isotope labeling. J Am Chem Soc 130: 16757-16769.

Kochoyan M, Lancelot G, Leroy JL. 1988. Study of structure, basepair opening kinetics, and proton exchange mechanism of the d-(AATTGCAATT) self-complementary oligodeoxynucleotide in solution. Nucleic Acids Res 16: 7685-7702.

Kozak M. 2005. Regulation of translation via mRNA structure in prokaryotes and eukaryotes. Gene 361: 13-37.

Leroy JL, Broseta D, Gueron M. 1985. Proton exchange and basepair kinetics of poly $(\mathrm{rA}) \bullet \operatorname{poly}(\mathrm{rU})$ and poly(rI)•poly(rC). J Mol Biol 184: 165-178.

Leroy JL, Kochoyan M, Huynh-Dinh T, Gueron M. 1988. Characterization of base-pair opening in deoxynucleotide duplexes using catalyzed exchange of the imino proton. J Mol Biol 200: 223238.

Lis H, Sharon N. 1998. Lectins: Carbohydrate-specific proteins that mediate cellular recognition. Chem Rev 98: 637-674.

Liu L, Yang C, Guo QX. 2000. A study on the enthalpy-entropy compensation in protein unfolding. Biophys Chem 84: 239-251.

Lumry R, Rajender S. 1970. Enthalpy-entropy compensation phenomena in water solutions of proteins and small molecules: A ubiquitous property of water. Biopolymers 9: 1125-1127.

Morse SE, Draper DE. 1995. Purine-purine mismatches in RNA helices: Evidence for protonated $\mathrm{G} \bullet \mathrm{A}$ pairs and next-nearest neighbor effects. Nucleic Acids Res 23: 302-306.

Nagel JH, Pleij CW. 2002. Self-induced structural switches in RNA. Biochimie 84: 913-923.

Olsen GL, Bardaro MF Jr, Echodu DC, Drobny GP, Varani G. 2010. Intermediate rate atomic trajectories of RNA by solid-state NMR spectroscopy. J Am Chem Soc 132: 303-308.

Patel DJ, Hilbers CW. 1975. Proton nuclear magnetic resonance investigations of fraying in double-stranded d-ApTpGpCpApT in $\mathrm{H}_{2} \mathrm{O}$ solution. Biochemistry 14: 2651-2656.

Reblova K, Spackova N, Stefl R, Csaszar K, Koca J, Leontis NB, Sponer J. 2003. Non-Watson-Crick base pairing and hydration in RNA motifs: Molecular dynamics of 5S rRNA loop E. Biophys J 84: 3564-3582.

Rinnenthal J, Klinkert B, Narberhaus F, Schwalbe H. 2010. Direct observation of the temperature-induced melting process of the Salmonella fourU RNA thermometer at base-pair resolution. Nucleic Acids Res 38: 3834-3847.

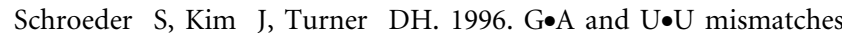
can stabilize RNA internal loops of three nucleotides. Biochemistry 35: $16105-16109$.

Sharp K. 2001. Entropy-enthalpy compensation: Fact or artifact? Protein Sci 10: 661-667.

Tinoco I Jr, Uhlenbeck OC, Levine MD. 1971. Estimation of secondary structure in ribonucleic acids. Nature 230: 362-367.

Treiber DK, Williamson JR. 2001. Beyond kinetic traps in RNA folding. Curr Opin Struct Biol 11: 309-314.

Varani G, Wimberly B, Tinoco I Jr. 1989. Conformation and dynamics of an RNA internal loop. Biochemistry 28: 77607772.

Watts JM, Dang KK, Gorelick RJ, Leonard CW, Bess JW Jr, Swanstrom R, Burch CL, Weeks KM. 2009. Architecture and secondary structure of an entire HIV-1 RNA genome. Nature 460: 711-716.

Woodside MT, Anthony PC, Behnke-Parks WM, Larizadeh K, Herschlag D, Block SM. 2006. Direct measurement of the full, sequence-dependent folding landscape of a nucleic acid. Science 314: 1001-1004.

Zhang Q, Stelzer AC, Fisher CK, Al-Hashimi HM. 2007. Visualizing spatially correlated dynamics that directs RNA conformational transitions. Nature 450: 1263-1267. 

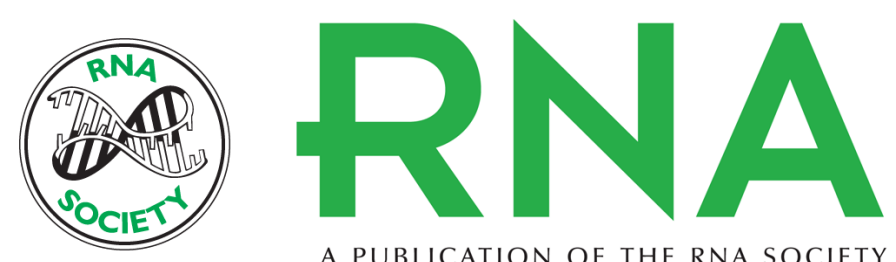

A PUBLICATION OF THE RNA SOCIETY

\section{Thermodynamics of RNA melting, one base pair at a time}

Evgenia N. Nikolova and Hashim M. Al-Hashimi

RNA 2010 16: 1687-1691 originally published online July 21, 2010

Access the most recent version at doi:10.1261/rna.2235010

\section{References This article cites 40 articles, 4 of which can be accessed free at: http://rnajournal.cshlp.org/content/16/9/1687.full.html\#ref-list-1}

\section{License}

Email Alerting Receive free email alerts when new articles cite this article - sign up in the box at the Service top right corner of the article or click here. 